\title{
Dense but confined matter as a new state in QCD
}

\section{Kurt Langfeld ${ }^{* *}$}

School of Computing \& Mathematics, University of Plymouth, Plymouth PL4 8AA, UK

E-mail: kurt.langfeld@plymouth.ac.uk

Centre sector transitions in QCD-like theories with dynamical quark matter are investigated. In the hadronic phase, these transitions still take place in the infinite volume at zero temperature limit despite of the explicit breaking of the centre symmetry by the matter fields. This finding is supported by simulations of the SU(2) Yang-Mills theory with Higgs matter. The phenomenological impact of the centre sector transitions for dense but confined matter is explained: centre dressed quarks acquire Bose statistics and form a so-called Fermi-Einstein condensate. This mechanism is further illustrated in the Schwinger model where it solves the Silver-Blaze problem.

International Workshop on QCD Green's Functions, Confinement and Phenomenology, September 05-09, 2011

Trento Italy

\footnotetext{
* Speaker.

${ }^{\dagger}$ It is a pleasure to thank Andreas Wipf for the collaboration on this project.
} 


\section{Introduction:}

Under normal conditions, eventually the most prominent feature of hadronic matter is colour confinement: quarks merely act as constituents of hadrons and qualify as auxiliary fields in the sense that the QCD partition function is solely given in terms of states with $N$-ality zero. Supposedly, the situation changes under extreme conditions, temperature and/or density, where quarks and gluons are liberated. This picture is corroborated at zero density and high temperatures by means of lattice Monte-Carlo simulations, which offer a first principle approach with controllable error margins. The picture is clear-cut in the heavy quark limit: the hadronic phase is characterised by a Wigner-Weyl realisation of the centre symmetry while the so-called gluon plasma phase at high temperature relates to a spontaneously breaking of this symmetry [1, 2]. Quite recently [3, 4], it was argued that this picture extends to the theory with dynamical quarks as well: although centre symmetry is explicitly broken by the quark matter, centre sector transitions do still take place in the hadronic phase [3] (see below for further details). The role of the quarks here is to merely induce a bias towards the trivial centre sector. Only at high temperature, the centre symmetry also breaks spontaneously giving rise to the quark gluon plasma phase.

The properties of cold, but dense QCD matter is far less understood due to the infamous signproblem which prevents Monte-Carlo simulations based upon Importance Sampling. A promising attempt to reach beyond the scope of Importance Sampling and to simulate QCD in this region of the phase diagram resorts to Complex Langevin dynamics [5] though further studies are needed to understand their convergence properties $[6,7]$. The intuition on dense fermionic matter arises from the free Fermi gas model: Antiperiodic boundary conditions in (Euclidean) time direction imply the formation of a Fermi sphere for finite chemical potential. The spin-statistics theorem $[8,9]$ ties particles with half-integer spin to fermion statistics. Note, however, that the theorem requires propagating (finite mass) particles with a positive definite norm. The statistics of confined quarks is therefore not necessarily restricted by the spin-statistics theorem. In fact, it was shown in [10] that in the confinement regime a certain "large" change of the gluon background field can be traded in for a change of the quark boundary conditions [10]. Another well known example originates from QCD perturbation theory: fermionic ghost fields inherit periodic boundary conditions from the gluon sector and evade the spin-statistics theorem since they involve negative norm states. The observation that quarks do not necessarily comply with anti-periodic boundary conditions, but, at least for an even number of colours, might acquire Bose statistics has a tremendous phenomenological impact: if, by virtue of the gluonic background, quarks develop a Matsubara zero mode, they might underdo condensation if the chemical potential equals the quark mass gap. This phenomenon has been called Fermi-Einstein condensation [11, 3, 4].

Considering confined quarks as auxiliary fields, a constraint to thermodynamical observables emerge form the sheer fact that the sole excitations are provided by hadrons: at zero temperature, observables are independent of the chemical potential as long as it is smaller than the hadronic mass gap. A widespread problem is that approximate methods and QCD models fail to comply with this physical fact. Accordingly, this problem has been called the Silver Blaze problem by Cohen in [12] reminiscent of the corresponding short story by Sir Arthur Conan Doyle, in which a dog doing nothing provides the essential clue to solve the crime mystery. 

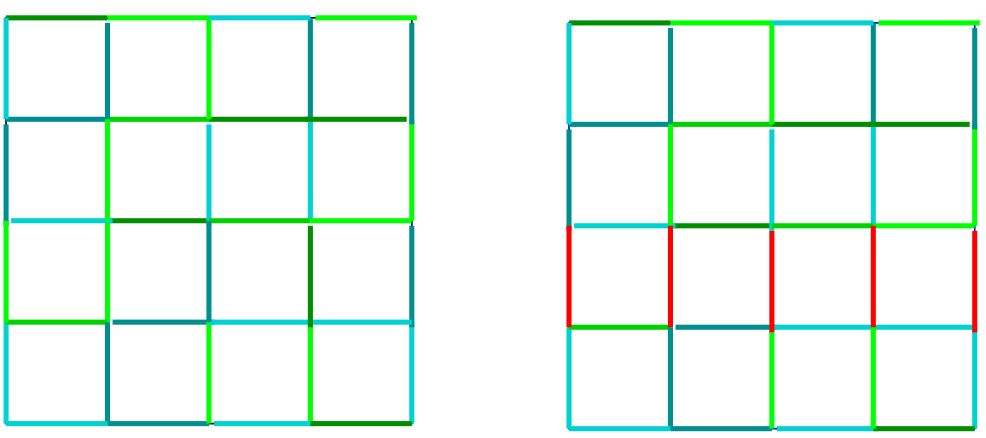

Figure 1: Sketch of a centre sector transition from a generic gluonic lattice configuration (left) to its centre transform (right).

\section{Centre sector transitions}

Let us adopt the lattice notation to illustrate the impact of centre sector transitions. A generic gluon configuration is given in terms of the links $U_{\mu}(x) \in S U\left(N_{c}\right)$ and is illustrated in figure 1, left panel. A particular Polyakov line is defined by

$$
P(\vec{x})=\frac{1}{N_{c}} \operatorname{tr} \prod_{x_{0}=1}^{N_{c}} U_{0}\left(x_{0}, \vec{x}\right)
$$

A centre transformed configuration is generated by multiplying the time-like links of a particular time slice $t$ by a centre element $Z_{n}=z_{n} 1 \in S U\left(N_{c}\right)$ :

$$
U_{0}^{(n)}(t, \vec{x})=Z_{n} U_{0}(t, \vec{x}), \quad \forall \vec{x}, \quad U_{\mu}^{(n)}(x)=U_{\mu}(x) \quad \text { else. }
$$

It is easy to see that any lattice action $A$ of pure Yang-Mills theory, which is built upon closed contractible loops, is invariant under the transformation (2.2) while the Polyakov loop is not:

$$
A\left[U_{\mu}\right]=A\left[U_{\mu}^{(n)}\right], \quad P\left[U_{\mu}^{(n)}\right](\vec{x})=z_{n} P\left[U_{\mu}\right](\vec{x}) \quad z_{n}=\exp \left\{i \frac{2 \pi}{N_{c}} n\right\}, n=1 \ldots N_{c} .
$$

Using the spatial Polyakov loop average $p$, it is straightforward to associate a so-called centre sector to each lattice configuration:

$$
p=\frac{1}{V} \sum_{\vec{x}} P(\vec{x}), \quad C(p)=n, \quad n:\left|\arg (p)-\frac{2 \pi n}{N_{c}}\right| \rightarrow \min
$$

where

$$
\arg (p)=\varphi \in] 0,2 \pi], \quad p=|p| \exp \{\mathrm{i} \varphi\} .
$$

It is straightforward to show that the centre transformation (2.2) shifts the centre sector by $n$ :

$$
C\left(p\left[U_{\mu}\right]\right)=m \quad \rightarrow \quad C\left(p\left[U_{\mu}^{(n)}\right]\right)=m+n .
$$

It is the symmetry which is spanned by the transformation (2.2) which is spontaneously broken in high temperature pure Yang-Mills theory [1,2]. The phenomenon related to this breakdown is deconfinement: the static quark antiquark potential is no longer linearly rising at large distances, 


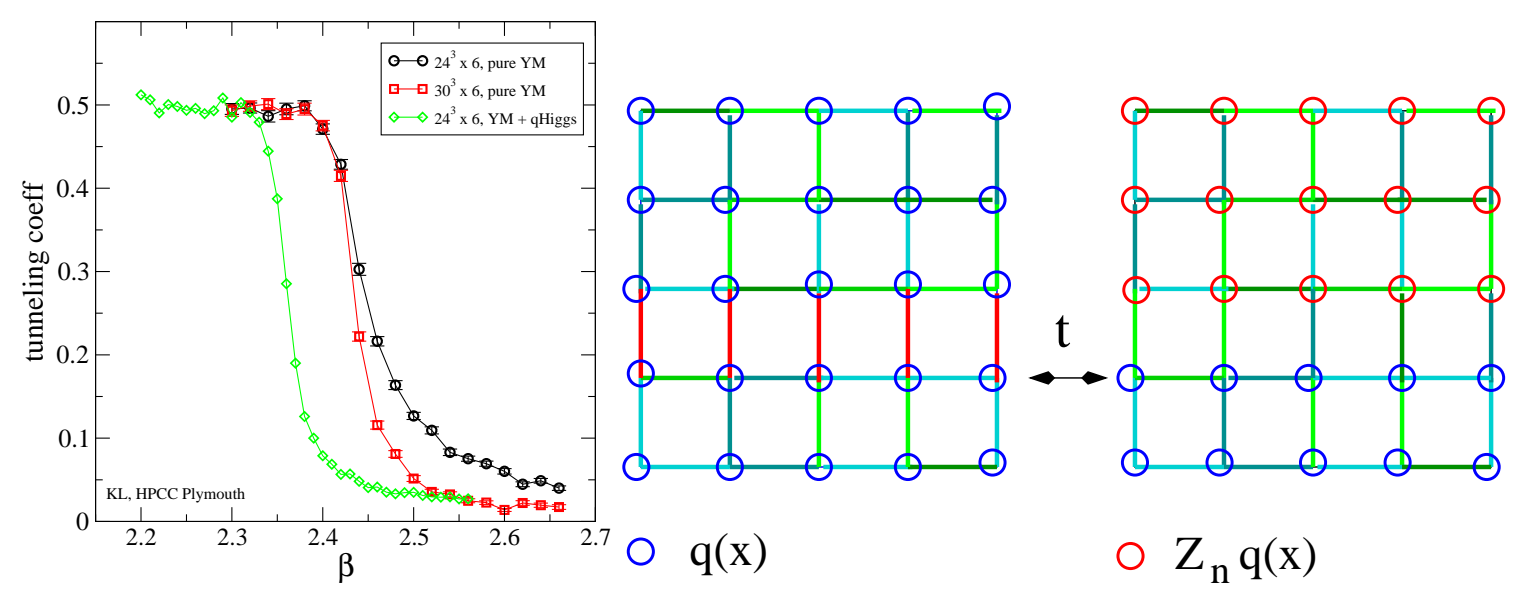

Figure 2: Right: Sector transition probability $\tau$ for the pure SU(2) YM-theory and the SU(2)-Higgs theory. Left: Roberge-Weisz transformation undoing a gluonic centre sector transition.

and the gluons contribute black body radiation to the thermal energy density suggesting gluon liberation.

How does this picture change for QCD-like theories, i.e., for gauge theories with dynamical matter which transforms under the fundamental representation of the gauge group? Let us consider for the moment $S U\left(N_{c}\right)$ Yang-Mills equipped with a quark determinant arising from integrating over the dynamical quark fields. The transformation (2.2) is no longer a symmetry of the action since the quark determinant provides a bias towards the trivial centre sector $n=N_{c}$. The question here is whether the lattice configurations are confined to the trivial centre sector because of this explicit centre symmetry breaking:

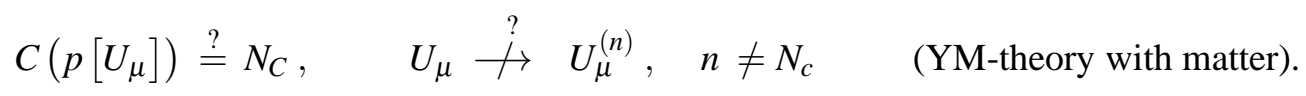

This question was thoroughly studied in [3] for the SU(2)-Higgs gauge theory for which the Higgs field plays the role of the dynamical matter. To detect whether centre sector transitions do occur, we divide the spatial lattice universe into two parts and calculate the spatially averaged Polyakov line over each part. Let us call the result $p_{<}$and $p_{>}$. Equally well, we can associate a centre sector to each half of the lattice universe by the mapping $C\left(p_{<}\right)$and $C\left(p_{>}\right)$. We then consider the probability $\tau$ that both centre sectors are different, i.e., $C\left(p_{<}\right) \neq C\left(p_{>}\right)$. If centre sector transitions do occur, $C\left(p_{>}\right)$and $C\left(p_{<}\right)$roughly sample all sectors ranging from $1 \ldots N_{c}$ leaving us with $\tau \approx 1-1 / N_{c}$. If, on the other hand, centre sector transitions are prohibited, we would have $\tau=0$. The so-called "tunnelling coefficient" $\tau$ is shown in figure 2, right panel, for the pure SU(2) Yang-Mills theory as function of the Wilson- $\beta$ parameter. While $\tau$ is close to $1 / 2$ at low temperatures, it rapidly drops for large $\beta$ values, which correspond to the gluon plasma phase. Also shown are the findings for the $\mathrm{SU}(2)$ Higgs theory: note that $\tau$ is still close to $1 / 2$ at small $\beta$ indicating that centre sector transitions do frequently occur. It is only above a certain critical value when these transitions cease to exist due to a spontaneous breaking of the centre symmetry besides of its explicit breaking. Also note that the critical $\beta$ value for deconfinement is smaller for the Higgs theory than for the pure 
YM-theory. This shows that the deconfinement critical temperature is smaller for the Highs theory, which is expected due to the matter effects.

Let us now include dynamical quark matter $q(x)$ which is subjected to a centre transformed gluon background field $U_{\mu}^{(n)}(2.2)$ (see figure 2, right panel). The matter gluon interaction is assumed to be of next-to-nearest-neighbour type: $\bar{q}(x) U_{\mu}(x) q(x+\mu)$. It turns out [10] that the centre transformation of the gluon fields can be reversed by transforming the quarks fields via

$$
q^{(n)}(x)=Z_{n} q(x) \text { for } x_{0}>t, \quad q^{(n)}(x)=q(x) \text { else. }
$$

This almost appears to be just a change of variables and therefore an invariance of the partition function. Note, however, that with the transformation (2.4) the boundary conditions of the quarks change from antiperiodic to $Z_{n}$-periodic:

$$
q\left(x_{0} 1 / T, \vec{x}\right)=(-1) Z_{n} q\left(x_{0}, \vec{x}\right)
$$

Let us $\operatorname{Det}_{\mathrm{AP}}$ denote the quark determinant with antiperiodic boundary conditions and let $\operatorname{Det}_{(n)}$ represent the determinant with $Z_{n}$-periodic boundary conditions. Since the partition function of QCD (or the QCD-like theory) is a gluonic ensemble average over all centre sectors, the partition function can be written as

$$
\int \mathscr{D} U_{\mu} \operatorname{Det}_{\mathrm{AP}}\left[U_{\mu}\right] \exp \left\{-S_{\mathrm{YM}}\left[U_{\mu}\right]\right\}=\int \mathscr{D} U_{\mu}^{\left(N_{c}\right)}\left(\sum_{n} \operatorname{Det}_{(n)}\left[U_{\mu}^{\left(N_{c}\right)}\right]\right) \exp \left\{-S_{\mathrm{YM}}\left[U_{\mu}^{\left(N_{c}\right)}\right]\right\} .
$$

Note that for an even number of colours, there is the centre element $Z_{N_{c} / 2}=-1$. Hence, there is a particular centre sector, which gives rise to periodic boundary conditions for the quark determinant when the gluon fields are mapped to the trivial sector by means of the transformation (2.4). In zeroth order perturbation theory, i.e., for $U_{\mu}^{\left(N_{c}\right)}=1$, the right hand side of (2.5) given rise to a centre-symmetric Fermi gas model. For $N_{c}$ even, it was shown in [10] that when the chemical potential $\mu$ approaches the mass gap $m$, the baryon density $\rho$ is largely determined by the centre sector $n=N_{c} / 2$ alone:

$$
\rho \approx \int_{m} d E \frac{-1}{\mathrm{e}^{[E-\mu] / T}-1}, \quad\left(N_{c} \text { even, confinement phase }\right) .
$$

where $E$ can be interpreted as the one-particle energy of the (modified) Fermi gas model. Notably, this contribution is singular for $\mu \rightarrow m$, and suggests an instability due to condensation quite analogously to Bose-Einstein condensation. Since the degrees of freedom which condense are fermions which are exposed to a non-trivial centre background field, this has been called Fermi-Einstein condensation (FEC). It is important to note that FEC can only occur in the confined phase: in this phase, transitions from the trivial centre sector $n=N_{c}$ to the sector with $n=N_{c} / 2$ do occur. Under extreme conditions, this is no longer the case: the sector transitions cease to exist, and only the trivial centre sector background is attained. In this case, the above model coincides with the standard Fermi gas model displaying Fermi statistics:

$$
\rho \approx \int_{m} d E \frac{1}{\mathrm{e}^{[E-\mu] / T}+1}, \quad \text { (high temperature deconfinement phase). }
$$


The question arises whether FEC also takes place in QCD-like theories with an odd numbers of colours and, most importantly, in QCD. To gain first insights, the centre symmetric quark model, i.e., zeroth order perturbation theory, has been generalised to the SU(3) gauge group [3]. The phase diagram can be analytically calculated in this model. It was found that, for low temperature and intermediate values of the chemical potential, a phase with an excess of baryon density does occur if the spatial volume is small enough, i.e., if the system is under pressure [3].

\section{Lessons from the Schwinger model}

The Schwinger model [13], i.e., QED in two dimensions on a space-time torus, is an ideal testbed for tracing out new ideas since many computations can be done analytically. Here, we will study the phenomenology of the centre-sector transitions in this model for a non-vanishing chemical potential.

The model with massless fermions was exactly solved in Hamiltonian formalism on the line in $[14,15,16]$ and on $S^{1}$ in $[17,18]$. The model on the torus has been studied in [19] and in particular the temperature dependence of the chiral condensate, Wilson loop correlators and Polyakov line correlators have been determined [20,21, 22]. In turns out that chiral symmetry is spontaneously broken and that only states with a vanishing net baryon number appear in the spectrum. The latter observation is key and implies that the baryon density ought to vanish even in the case of nonvanishing values of the chemical potential. Non-vanishing values of the fermion chemical potential have been firstly considered in [23, 24], and it was found, indeed, that the full non-perturbative partition function is independent of the chemical potential.

The gauge potential can be written as [20]

$$
A_{0}=\frac{2 \pi}{\beta} h_{0}+\partial_{0} \lambda-\partial_{1} \phi, \quad A_{1}=\frac{2 \pi}{L} h_{1}+\partial_{1} \lambda+\partial_{0} \phi
$$

where the periodic functions $\lambda$ and $\phi$ integrate to zero. The constant toron fields $h_{0,1} \in[0,1[$ label the U(1) centre sectors of the model. This can be easily seen from the fact that the shift $h_{0} \rightarrow h_{0}+\alpha$ transforms the Polyakov line by a $\mathrm{U}(1)$ centre element $P(x) \rightarrow \exp \{\mathrm{i} 2 \pi \alpha\} P(x)$. The partition function factorise into a photonic part and into the centre sector average of the fermion determinant:

$$
Z(T, L, \mu)=(2 \pi)^{2} \sqrt{\frac{\operatorname{det}^{\prime}(-\triangle)}{\operatorname{det}^{\prime}\left(-\triangle+m_{\gamma}^{2}\right)}} \int_{0}^{1} d h_{0} d h_{1} \operatorname{det}\left(\mathrm{i} \not_{h, \mu}\right),
$$

where $m_{\gamma}$ is a dynamically generated photon mass, and $L$ is the spatial extent of the torus. Assuming a frozen centre sector, i.e., a fixed value for $h_{0}$, would lead to a Silver Blaze problem since the baryon density $\rho_{B}$ is non-vanishing in this case:

$$
\rho_{B} \stackrel{L \rightarrow \infty}{\longrightarrow} \frac{1}{\pi} \int_{0}^{\infty} d p\left\{\frac{z}{\mathrm{e}^{\beta(p-\mu)}+z}-\frac{z^{*}}{\mathrm{e}^{\beta(p+\mu)}+z^{*}}\right\}, \quad z=\exp \left\{-2 \pi \mathrm{i} h_{0}\right\} .
$$

Note also that for the particular choice $h_{0}=1 / 2$, we find $z=-1$ implying that the one-particle distribution functions are of Bose type. Thus, freezing the centre sector yields the wrong physics. 
On the other hand, the centre sector average, i.e., the integration over the toron field $h_{0}$, yields a result which is independent of $\mu$ [3], i.e.,

$$
Z(T, L, \mu)=\sqrt{\frac{V}{2}} \frac{1}{\sqrt{\operatorname{det}^{\prime}\left(-\triangle+m_{\gamma}^{2}\right)}},
$$

and, thus, solves the Schwinger-Blaze problem in the Schwinger model.

\section{References}

[1] B. Svetitsky, L. G. Yaffe, Nucl. Phys. B210 (1982) 423.

[2] B. Svetitsky, Phys. Rept. 132 (1986) 1-53.

[3] K. Langfeld and A. Wipf, Fermi-Einstein condensation in dense QCD-like theories, arXiv:1109.0502 [hep-lat], accepted by Annals of Physics.

[4] K. Langfeld and A. Wipf, From confinement to new states of dense QCD matter, arXiv:1111.4315 [hep-lat], in press by Prog. Part. Nucl. Phys.

[5] G. Parisi, Phys. Lett. B 131 (1983) 393.

[6] G. Aarts, E. Seiler and I. -O. Stamatescu, Phys. Rev. D 81 (2010) 054508 [arXiv:0912.3360 [hep-lat]].

[7] G. Aarts, F. A. James, E. Seiler and I. -O. Stamatescu, Phys. Lett. B 687 (2010) 154 [arXiv:0912.0617 [hep-lat]].

[8] W. Pauli, Phys. Rev. 58 (1940) 716.

[9] Steven Weinberg, The Quantum Theory of Fields: Volume 1, Foundations, Cambridge University Press 1995.

[10] K. Langfeld, B. H. Wellegehausen and A. Wipf, Phys. Rev. D 81 (2010) 114502 [arXiv:0906.5554 [hep-lat]].

[11] K. Langfeld, PoSQCD -TNT09 (2009) 022 [arXiv:0911.0319 [hep-lat]].

[12] T. D. Cohen, Phys. Rev. Lett. 91 (2003) 222001 [arXiv:hep-ph/0307089].

[13] J. S. Schwinger, Phys. Rev. 128 (1962) 2425-2429.

[14] L.S. Brown, Nuovo Cimento 29 (1963) 617-643

[15] J. Lowenstein, A. Swieca. Ann. Phys. 68 (1971) 172-195;

[16] A. Casher, J. B. Kogut, L. Susskind, Phys. Rev. D10 (1974) 732-745.

[17] N. Manton, Ann. Phys. 159 (1985) 220-251

[18] S. Iso, H. Murayama, Prog. Theor. Phys. 84 (1990) 142-163.

[19] H. Joos, Helv. Phys. Acta 63 (1990) 670-682.

[20] I. Sachs and A. Wipf, Helv. Phys. Acta 65 (1992) 652-678. [arXiv:hep-th/1005.1822]

[21] A. V. Smilga, Phys. Lett. B278 (1992) 371-376.

[22] S. Azakov, Fortsch. Phys. 45 (1997) 589-626. [hep-th/9608103].

[23] I. Sachs and A. Wipf, Annals Phys. 249 (1996) 380-429. [arXiv:hep-th/9508142]

[24] R. F. Alvarez-Estrada, A. Gomez Nicola, Phys. Rev. D57 (1998) 3618-3633. [hep-th/9710227]. 